\title{
Tracking crystallophore nucleating properties: setting-up a database for statistical analysis.
}

Tao Jiang 1, Amandine Roux 1§, Sylvain Engilberge 2†, Zaynab Alsalman 2, Sebastiano Di

Pietro 1\#, Bruno Franzetti 2, François Riobé 1*, Olivier Maury 1*, Eric Girard 2*.

1 Univ. Lyon, ENS de Lyon, CNRS UMR 5182, Université Claude Bernard Lyon 1, Laboratoire de Chimie, F69342, Lyon, France;

2 Univ. Grenoble Alpes, CEA, CNRS, Institut de Biologie Structurale, 38000 Grenoble, France;

$\S$ Present address: Polyvalan SAS, Lyon, France

$\dagger$ Present address: Swiss Light Source, Paul Scherrer Institute, CH-5232 Villigen PSI,

Switzerland

\# Present address: Dipartimento di Farmacia, Università di Pisa, Via Bonanno 33, 56126 Pisa, Italy

* Correspondence: eric.girard@ibs.fr; olivier.maury@ens-lyon.fr; francois.riobe@ens-lyon.fr

KEYWORDS: Crystallophore, Nucleating agent, Molecular glues, Protein crystallization, Data mining, Subset-of-interest. 


\begin{abstract}
In this article, the principle of a database aimed at facilitating the understanding of the unique protein nucleating properties of the Crystallophore is presented. A first analysis allows us to compare the efficiency of $\mathrm{Tb}-\mathrm{Xo} 4$ with the new Lu-Xo4 variant, featuring improved phasing properties. Then, the concept of subset-of-interest is introduced to reveal potential antagonistic/synergistic effects between $\mathrm{Tb}-\mathrm{Xo} 4$ and physico-chemical parameters of the crystallisation kits such as $\mathrm{pH}$. The overall approach may be of interest for any studies working on solutions dedicated to improve the nucleating step in protein crystallization.
\end{abstract}

\title{
TOC Graphic:
}

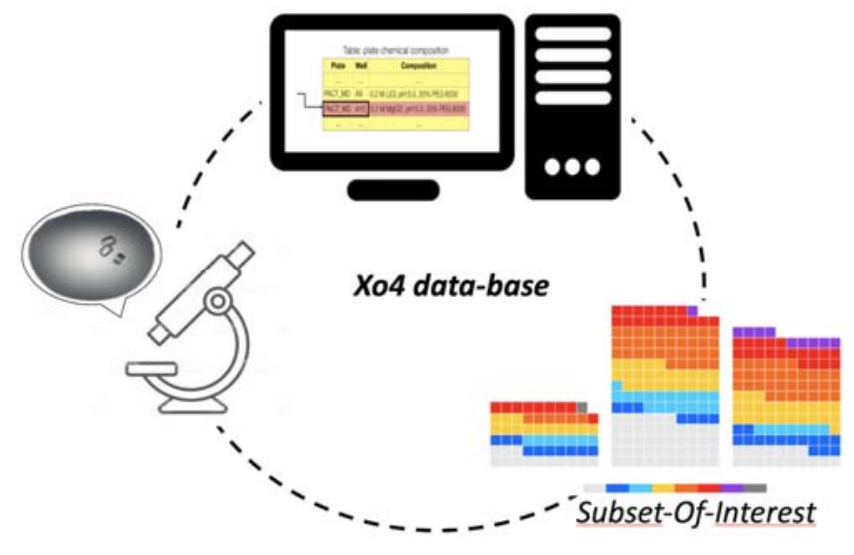

Synopsis: A database and associated representation tools are highlighted to understand nucleating properties of the crystallophore. 


\section{INTRODUCTION}

The success of protein structure determination by X-ray crystallography obviously relies on the availability of well-diffracting crystal that remains precisely the major hurdle in this multi-steps process. Automation associated with the use of nano-volume dispensing robot has tentatively increased the efficiency of the process. Depending on protein sample availability, hundred to thousand crystallization conditions can be evaluated thanks to the various commercial kits available on the market, without guarantee of success in getting a suitable condition. Different approaches facilitating the nucleation step have been proposed: (i) when an initial crystallization condition provides even poor quality crystals or related like urchins, homogenous nucleation can be envisaged through seeding of crystallization drops with fragments of crystal. ${ }^{1-4}$ (ii) Secondly, heterologous nucleation relies on the introduction of materials within the drop at the start of the crystallization process. Various materials have been evaluated with more or less success. ${ }^{5-11}$ (iii) Finally, the recent development of molecular glues (calixarene, polyoxometalate or lanthanide complexes) able to improve or mediate the protein-protein contacts is nowadays the most straightforward strategy to improve the nucleation step. ${ }^{12-17}$ Among these molecular glues we introduced, in 2017, the crystallophore. ${ }^{18}$

The crystallophore, Xo4, is a family of cationic lanthanide complexes presenting both nucleating and phasing properties in an all-in-one tool which is capable of overcoming the two main bottlenecks of macromolecular crystallography. ${ }^{18}$ The story so far of the Xo4 system started with its terbium variant $\mathrm{Tb}$-Xo4. Its unique properties were firstly highlighted with a set of 8 proteins and its efficiency was confirmed in solving issues often dealt by crystallographer in their quest to assess the protein structure. ${ }^{18,19}$ Finally, Tb-Xo4 was exploited to determine new protein structures including multi-protein complexes ${ }^{20-22}$ and can be used as a routine nucleating and phasing tool by the community. ${ }^{23-27}$

To understand the origins of the crystallophore nucleating and phasing properties with the aim to further improve them, we analyzed the binding of $\mathrm{Tb}-\mathrm{Xo} 4$ at the surface of proteins by coupling crystal structure analysis, obtained on 4 different proteins, with evaluation of the interaction energies determined by density functional theory calculations. ${ }^{28}$ This detailed analysis of the interaction sites on the proteins surfaces clearly demonstrated the great versatility of $\mathrm{Tb}$ Xo4 binding through various supramolecular interactions via anionic, cationic or hydrophobic amino-acid residues. Such versatility may explain the unprecedented properties of this 
compound. However, this variability in the interaction mode from one example to another prevents any determination of an optimal amino-acid environment required for an efficient interaction of $\mathrm{Tb}-\mathrm{Xo} 4$ that could enhance the nucleation process. Furthermore, the study also revealed the non-negligible role of the particular composition of the crystallization solution or protein buffer in the global interaction networks. We observed the direct involvement of components, such as $\mathrm{Ca}^{2+}$ cation or glycerol molecule, in the interaction network. Sulfate or iodide anions may also directly interact with crystallophore turning its overall charge (monocationic) into a neutral or even an anionic one thus modulating the interaction energies balance. ${ }^{28}$ Thus, it appears clearly that the crystallization solution components may directly participate to the nucleation process making its study much more complicated due to the huge number of independent parameters.

In order to understand how crystallization kits formulation and corresponding physicochemical conditions may influence the nucleating effect of the crystallophore, we set-up a database to efficiently analyze the influence of crystallization media components. In the present paper, we present the structure of this database through a first analysis of 6 crystallization trials that were performed in the course of our previous work. ${ }^{18}$ This analysis will benefit from the comparison with the crystallization screening in native conditions that was run in absence of the crystallophore. These 6 proteins (hen egg white lysozyme (HEWL), Tritirachium album proteinaseK, Thaumatococcus daniellii thaumatin, protease one from P. horokoshii, Glyoxylate Hydroxypyruvate Reductase from Pyrococcus furiosus (GRHPR) and bacteriophage T5 distal tail protein (pb9)) were evaluated with the same commercial crystallization kits. ${ }^{18}$

Lanthanide complexes chemistry also offers the possibility to explore several variants of the crystallophore obtained by chemical modifications of the Xo4 ligand, possibly altering interactions properties of the generated variant and thus nucleating properties. In the present work, we introduce a variant of the crystallophore, where the terbium (III) ion was substituted with lutetium (III) (Scheme 1). Indeed, based on a previous study, ${ }^{29}$ the lutetium crystallophore, Lu-Xo4, was synthetized in order to provide a phasing agent more convenient to use. The lutetium $\mathrm{L}_{\mathrm{III}}$ absorption edge, at $1.34 \AA$, is more easily accessible to beamlines dedicated to protein crystallography at synchrotron facilities (for comparison $\mathrm{L}_{\mathrm{III}}$ absorption edge of terbium is located at $1.65 \AA$ ). Indeed, when data resolution is limited by the sample environment, in particular the detector size and/or the crystal-to-detector distance, it may be advantageous to 
work at a shorter wavelength. Moreover, since anomalous effects are more important at high resolution, this gain facilitates the phasing step, making lutetium the most interesting lanthanide. Lutetium still conserves a large anomalous signal (with an anomalous contribution $f$ " of about 10 electrons) at the selenium $\mathrm{K}$ absorption edge and can be exploited for non-optimal anomalousbased experiment. It thus became clear to check if Lu-Xo4 retained the same nucleating properties as $\mathrm{Tb}-\mathrm{Xo} 4$.

Herein, we present the overall structure of the database and different representation modes of the information stored in it and their exploitation to compare, for example, the Tb-Xo4/Lu-Xo4 nucleating properties. In addition, exploiting the database to analyse the nucleating performance of $\mathrm{Tb}$-Xo4 even performed on a limited number of proteins also show a potential influence of the $\mathrm{pH}$ as well as a potential synergy between Salt-Grid (Hampton Research) and PACT (Molecular Dimensions) crystallization kits.

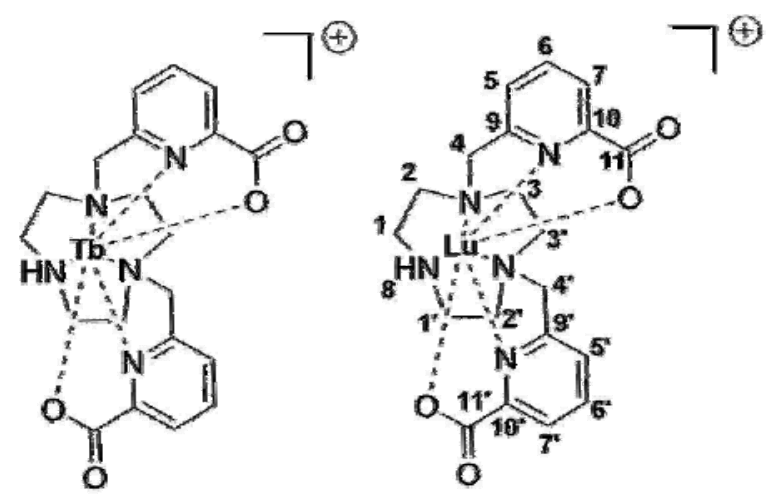

Scheme 1: Molecular structure of the crystallophore additives Tb-Xo4 and Lu-Xo4 with atom numbering.

\section{EXPERIMENTAL SECTION}

Crystallophore synthesis and purification. Tb-Xo4 and $\mathrm{Lu}-\mathrm{Xo} 4$ were produced using the same improved protocol. ${ }^{18}$ The acid ligand was formed in situ from $502 \mathrm{mg}$ of diester (1.17 mmol) in presence of $\mathrm{NaOH}$ (2 eq.) in a $\mathrm{H}_{2} \mathrm{O} / \mathrm{MeOH}$ mixture (9/1, v/v) stirred overnight at room temperature before the complexation step (HR-MS (ESI) calculated $\mathrm{m} / \mathrm{z}=400.1979$ $[\mathrm{M}+\mathrm{H}]^{+}$, measured $\mathrm{m} / \mathrm{z}=400.1975$ for $\mathrm{M}=\mathrm{C}_{20} \mathrm{H}_{26} \mathrm{~N}_{5} \mathrm{O}_{4}$ ). 
Then the crude acid ligand formed in situ was neutralized to $\mathrm{pH}=7$ adding $\mathrm{HCl}$ (1M) followed by the addition of $481 \mathrm{mg}$ of $\mathrm{TbCl}_{3} \cdot 6 \mathrm{H}_{2} \mathrm{O}(1.29 \mathrm{mmol}, 1.1 \mathrm{eq})$ to the mixture. The $\mathrm{pH}$ was increased until $\mathrm{pH}=5.5 \mathrm{using} \mathrm{NaOH}(1 \mathrm{M})$ and the resulting solution was stirred overnight at room temperature. After evaporation of the solvents, the Tb-Xo4 complex was purified using preparative HPLC separation. The purification was performed using a Nucleodur® (MachereyNagel) C18 HTec, $5 \mu \mathrm{m}$, Preparative VarioPrep $250 \mathrm{~mm}$ column at $5 \mathrm{~mL} / \mathrm{min}$. Mobile phase consisted in a gradient of solvent $\mathrm{A}\left(0.1 \%\right.$ ammonium formate in $\left.\mathrm{CH}_{3} \mathrm{CN}\right)$ and $\mathrm{B}(0.1 \%$ ammonium formate in $\mathrm{H}_{2} \mathrm{O}$ ). Method 1 was used: 5\% A during 2 min followed by a 5 to $100 \%$ A gradient in $14 \mathrm{~min}$ at $5 \mathrm{~mL} / \mathrm{min}$. Then the method carried on during 2 min with $100 \% \mathrm{~A}$ followed by a 100 to $5 \% \mathrm{~A}$ gradient in $8 \mathrm{~min}$. (Retention time $=17 \mathrm{~min}$ ). A white powder is obtained after freeze-drying with a yield of $78 \%(505 \mathrm{mg})$. HR-MS (ESI) calculated $\mathrm{m} / \mathrm{z}=$ $574.1104\left[\mathrm{M}+\mathrm{H}_{2} \mathrm{O}+\mathrm{H}\right]^{+}$, measured $\mathrm{m} / \mathrm{z}=574.1106$ for $\mathrm{M}=\mathrm{C}_{20} \mathrm{H}_{25} \mathrm{~N}_{5} \mathrm{O}_{5} \mathrm{~Tb}$.

The same protocol was used to synthesize the $\mathrm{Lu}-\mathrm{Xo} 4$ starting from $\mathrm{LuCl}_{3} \cdot 6 \mathrm{H}_{2} \mathrm{O}$ with a yield of 49\%. The preparative column used for HPLC purification was an Agilent ZORBAX SB-C18, $5 \mu \mathrm{m}, 100 \mathrm{~mm}$. The purification was done with the same Method 1 as for Tb-Xo4 (Retention time $=11 \mathrm{~min})$. HR-MS $(\mathrm{ESI})$ calculated $\mathrm{m} / \mathrm{z}=590.1258\left[\mathrm{M}+\mathrm{H}_{2} \mathrm{O}+\mathrm{H}\right]^{+}$, measured $\mathrm{m} / \mathrm{z}=$ 590.1239 for $\mathrm{M}=\mathrm{C}_{20} \mathrm{H}_{25} \mathrm{~N}_{5} \mathrm{O}_{5} \mathrm{Lu}$.

The NMR analysis (Scheme 1) is: ${ }^{1} \mathrm{H}-\mathrm{NMR}\left(300 \mathrm{MHz}, \mathrm{D}_{2} \mathrm{O}\right): \delta 8.31$ (dd, $\left.J=7.8 \mathrm{~Hz}, 1 \mathrm{H}, \mathrm{H} 6\right)$, 8.25 (dd, J= 7.8 Hz, 1H, H6'), 8.17 (d, J= 7.7 Hz, 1H, H5), 7.93 (d, J= 7.7 Hz, 1H, H5'), 7.86 (dd, J=7.2 Hz, 2H, H7/7'), 4.45 (AB system, $\delta_{\mathrm{A}}=4.51, \delta_{\mathrm{B}}=4.39, \mathrm{~J}_{\mathrm{AB}}=16 \mathrm{~Hz}, v_{\mathrm{A}}=1352 \mathrm{~Hz}$, $\left.v_{\mathrm{B}}=1315 \mathrm{~Hz}, 2 \mathrm{H}, \mathrm{H} 4\right), 4.25\left(\mathrm{AB}\right.$ system, $\delta_{\mathrm{A}}=4.29, \delta_{\mathrm{B}}=4.20, \mathrm{~J}_{\mathrm{AB}}=16 \mathrm{~Hz}, v_{\mathrm{A}}=1287 \mathrm{~Hz}$, $\left.v_{\mathrm{B}}=1261 \mathrm{~Hz}, 2 \mathrm{H}, \mathrm{H} 4^{\prime}\right), 4.12($ broad s, 1H, NH), 3.74 (t, J=12.9 Hz, 1H, H3'), 3.60 (m, 2H, H1'/H1), 3.41 (m, 1H, H3), 3.10 (t, J= 17.0 Hz, 1H, H3'), 3.09 (t, J=17.0 Hz, 1H, H3), 3.05 (m, 1H, H1'), 2.86 (m, 1H, H2'), 2.82 (m, 1H, H1), 2.59 (m, 2H, H2/H2'), 2.30 (t, J= 12.3 Hz, 1H, H2). ${ }^{13} \mathrm{C}-\mathrm{NMR}\left(100 \mathrm{MHz}, \mathrm{D}_{2} \mathrm{O}\right): \delta 172.4$ (C11), 172.1 (C11'), 158.4 (C10), 155.7 (C10'), 149.3 (C9), 148.6 (C9'), 142.8 (C6), 142.3 (C6’), 126.6 (C7), 126.4 (C7'), 124.1 (C5), 124.0 (C5'), 65.7 (C4), 64.3 (C4'), 57.0 (C3), 56.6 (C2), 54.7 (C3'), 54.0 (C1), 49.0 (C2'), 45.8 (C1').

Both complexes can be purchased from the company Polyvalan (Lyon, France).

Protein production and crystallization. Purchase, production and purification of the six proteins used in the present study (Table 1) as well as their crystallization using the Highthroughput Crystallization facility (HTXlab) at EMBL-Grenoble were previously described. ${ }^{18}$ In 
brief, each protein was evaluated in the absence and in the presence of $10 \mathrm{mM} \mathrm{Tb}-\mathrm{Xo} 4$ and crystallization assays were performed in 576 conditions from different commercial crystallization kits. Reported crystallization drop evaluation was performed at 90 days.

For the comparison of the nucleating properties of $\mathrm{Lu}-\mathrm{Xo} 4$ with $\mathrm{Tb}-\mathrm{Xo} 4$, crystallization assays with HEWL, ProteinaseK and Thaumatin were performed. The assays were run at the HTXlab similarly to the initial evaluation of Tb-Xo4 nucleating properties. ${ }^{18}$ Drop observation was performed after 15 days.

Crystallization conditions marked as hit correspond to drops containing well-defined crystals.

Table 1: Proteins used in this study and their properties.

\begin{tabular}{|c|c|c|c|c|c|}
\hline Protein & $\begin{array}{c}\text { Uniprot } \\
\text { entry }\end{array}$ & $\begin{array}{l}\text { No. of } \\
\text { residues }\end{array}$ & $\begin{array}{c}\text { Theoretical } \\
\text { pI }\end{array}$ & $\begin{array}{c}\text { No. of } \\
\text { Asp/Glu }\end{array}$ & $\begin{array}{l}\text { No. of } \\
\text { Lys/Arg }\end{array}$ \\
\hline HEWL & P00698 & 129 & 9.32 & 9 & 17 \\
\hline ProteinaseK ${ }^{1}$ & P06873 & 279 & 8.25 & 18 & 20 \\
\hline Thaumatin ${ }^{2}$ & P02883 & 207 & 8.46 & 18 & 23 \\
\hline Protease $1^{3}$ & O59413 & 166 & 6.11 & 24 & 22 \\
\hline GRHPR ${ }^{4}$ & Q8U3Y2 & 336 & 6.24 & 52 & 50 \\
\hline $\mathrm{pb}^{5}$ & Q6QGE8 & 217 & 6.15 & 23 & 20 \\
\hline
\end{tabular}

Hydroxypyruvate Reductase from Pyrococcus furiosus. ${ }^{5}$ bacteriophage T5 distal tail protein.

Database. The data are managed in a SQLite database. The SQLite3 Python library was used to interface with SQLite database for easy data query and modification. Usage of Python scripts is exploited as the data obtained from the query can be easily analyzed with the assistance of the Numpy library. Various visual representations can be generated by utilizing the Matplotlib library.

\section{RESULTS AND DISCUSSION}

Database structure. The overall structure of the database is illustrated in Figure 1. The data are stored in two tables. The samples table stores the assays performed with the name of the protein 
and if the assay was performed in the absence or in the presence of the crystallophore, through the protein column which possesses the structure XXX_YYY with XXX is the considered protein and YYY refers to native (absence of crystallophore) or to the presence of crystallophore. The hit tracking results are stored in the samples table. The plate chemical composition table contains all the detailed information of all well components of the different commercial crystallization kits. As highlighted in Figure 1, both samples and plate chemical composition tables possess the columns Plate and Well. The two tables can be joined together via these two columns to generate on the fly, thanks to a python script, the protein crystallization condition table, from which any analysis of the effects of the chemical compositions on the crystallization process can be performed.

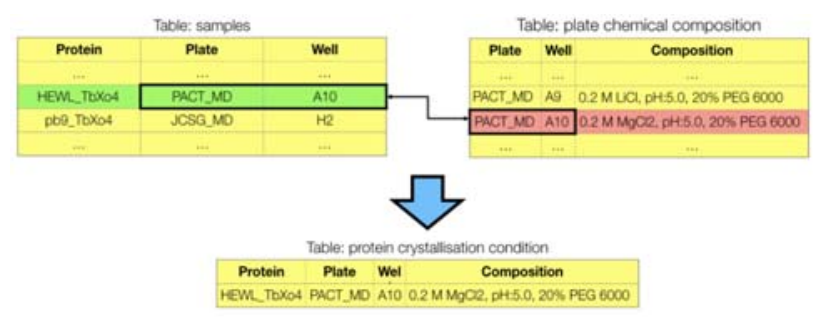

Figure 1: Overall representation of the database structure.

In the framework of studies devoted to understand the crystallization of biological macromolecules, the database offers the unique opportunity to perform rigorous comparative analysis by considering the crystallization of the native protein, i.e. in the absence of the crystallophore, as a control experiment and to explore the parameters leading to differences induced by Xo4 presence during the crystallization experiment. This exploration will be highly facilitated by the database as experienced by high-throughput crystallization facilities. Indeed, databases are at the heart of such facilities for their daily operation. These databases, as well as the Protein Data Bank, have also been exploited to determine the key factors that affect protein crystallization leading to provide optimized crystallization kits $\mathrm{kin}^{30-32}$ and to promote practical guidelines for initial screening experiments of new crystallization targets. ${ }^{33-35}$

By-plate representation. In our initial publication, ${ }^{18}$ results of crystallization assays were represented as a histogram providing the number of hits, i.e. the number of crystallization drops showing crystals, for the native protein (i.e. in the absence of $\mathrm{Tb}-\mathrm{Xo} 4$ ) and for the protein with 
Tb-Xo4 $(10 \mathrm{mM})$ as well as the number of hits observed for both native and Xo4-containing samples. This representation provides a raw view of the nucleating efficiency of the crystallophore (Figure 2a).

2.

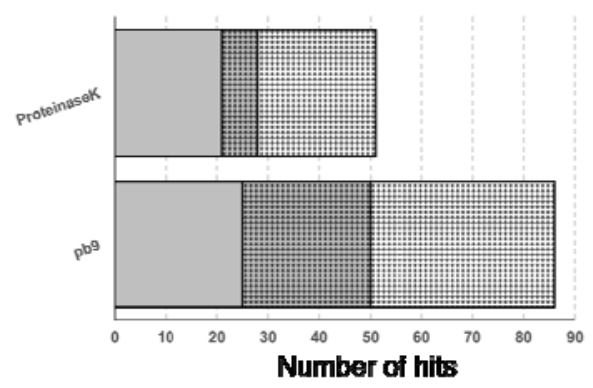

b

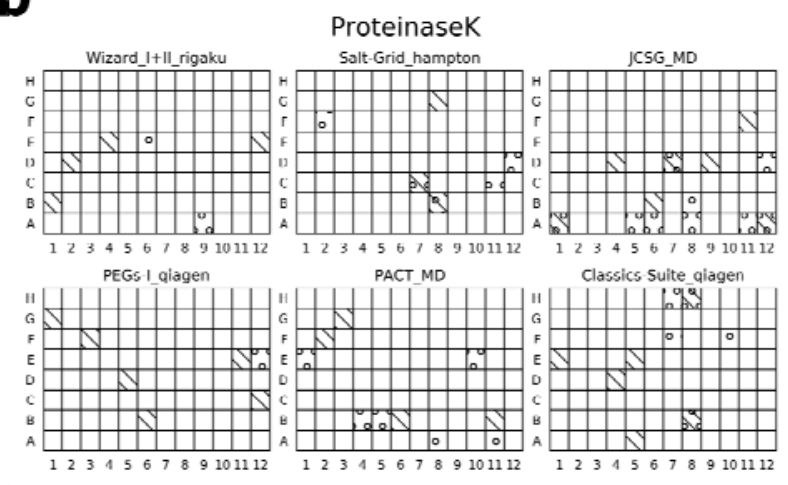

$\mathrm{pb} 9$
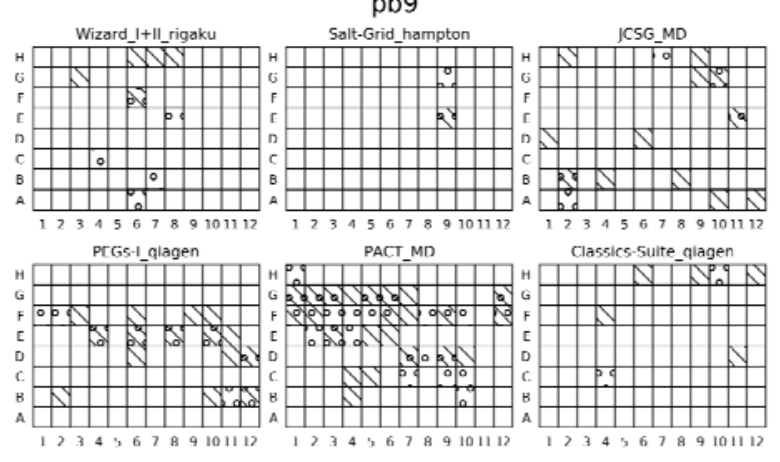

Figure 2: Various representations of the database information for the two proteins, ProteinaseK and pb9. (a) Histogram representation. The number of crystallization hits is depicted in grey for the native protein and with dots for protein supplemented with $10 \mathrm{mM}$ of Tb-Xo4. As a result, the common (shared) conditions are represented in grey with dots. (b) By-plate representations where hits without $\mathrm{Tb}-\mathrm{Xo} 4$ (native) are depicted by a circle while hits obtained in the presence of $10 \mathrm{mM}$ Tb-Xo4 are represented with a backslash. 
Thanks to the built database, we explore a by-plate representation (Figure 2b). This view allows a side-by-side comparison and also a direct identification of the conditions associated with the crystallization of the native protein alone or with the unique $\mathrm{Tb}-\mathrm{Xo} 4$ crystallization ones. This view may be of interest for a user looking at tendencies in term of crystallization components in the absence/presence of crystallophore to increase the chance to get exploitable crystals. Indeed, as illustrated in Figure $2 \mathrm{~b}$, complementary crystallization conditions can be directly identified and crystallization kits, that might have been neglected based on the native protein crystallization results, now show interest, such as the PEG-Ion or the Classic-Suite kits in the case of ProteinaseK and pb9, respectively.

Comparison of $\mathbf{L u - X o 4}$ variant with $\mathbf{T b}$-Xo4. We exploited the database and the by-plate representation to evaluate if $\mathrm{Lu}-\mathrm{Xo} 4$ variant retained the same nucleating properties as $\mathrm{Tb}-\mathrm{Xo} 4$. This was determined on three commercial proteins as described in the Experimental section and results.

As expected, most of the hits observed with Tb-Xo4 are recovered with Lu-Xo4 (Figure 3a). These common hits lead to crystals with similar habits (Figure 3b, common hits). However, we also observed crystallization conditions leading to crystals only in the presence of $\mathrm{Tb}-\mathrm{Xo} 4$ or only in the presence of Lu-Xo4. The number of these unique Tb-Xo4 hits is 18, 7 and 6 for HEWL, ProteinaseK and Thaumatin respectively, while it is 47, 18 and 6 for unique Lu-Xo4 hits. Illustrative drops are displayed in Figure 3b. If some of these unique hits corresponds to crystallization conditions close to those observed for common $\mathrm{Tb}-\mathrm{Xo} 4 / \mathrm{Lu}-\mathrm{Xo} 4$ hits or may be related to the variability commonly observed in crystallization assays, this does not fully explain the large number observed for Lu-Xo4 in the case of HEWL and ProteinaseK. This may reflect a slightly higher nucleating property of Lu-Xo4. Considering the prevalence of the binding of the central lanthanide ion with pendant carboxylate-containing amino-acids in the Ln-Xo4 interaction modes, this improvement could be induced by the stronger Lewis acidity of the lutetium(III) regards to terbium(III). 

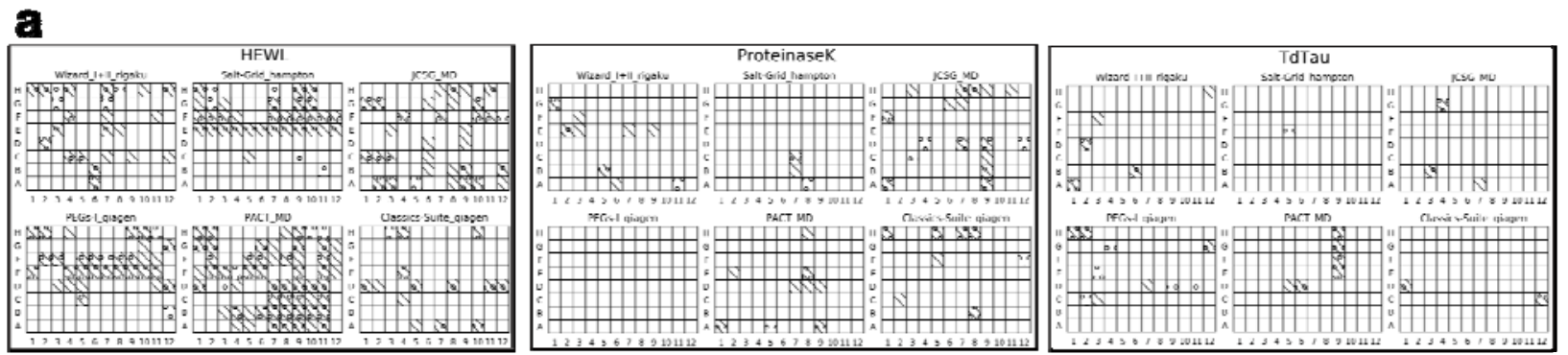

b
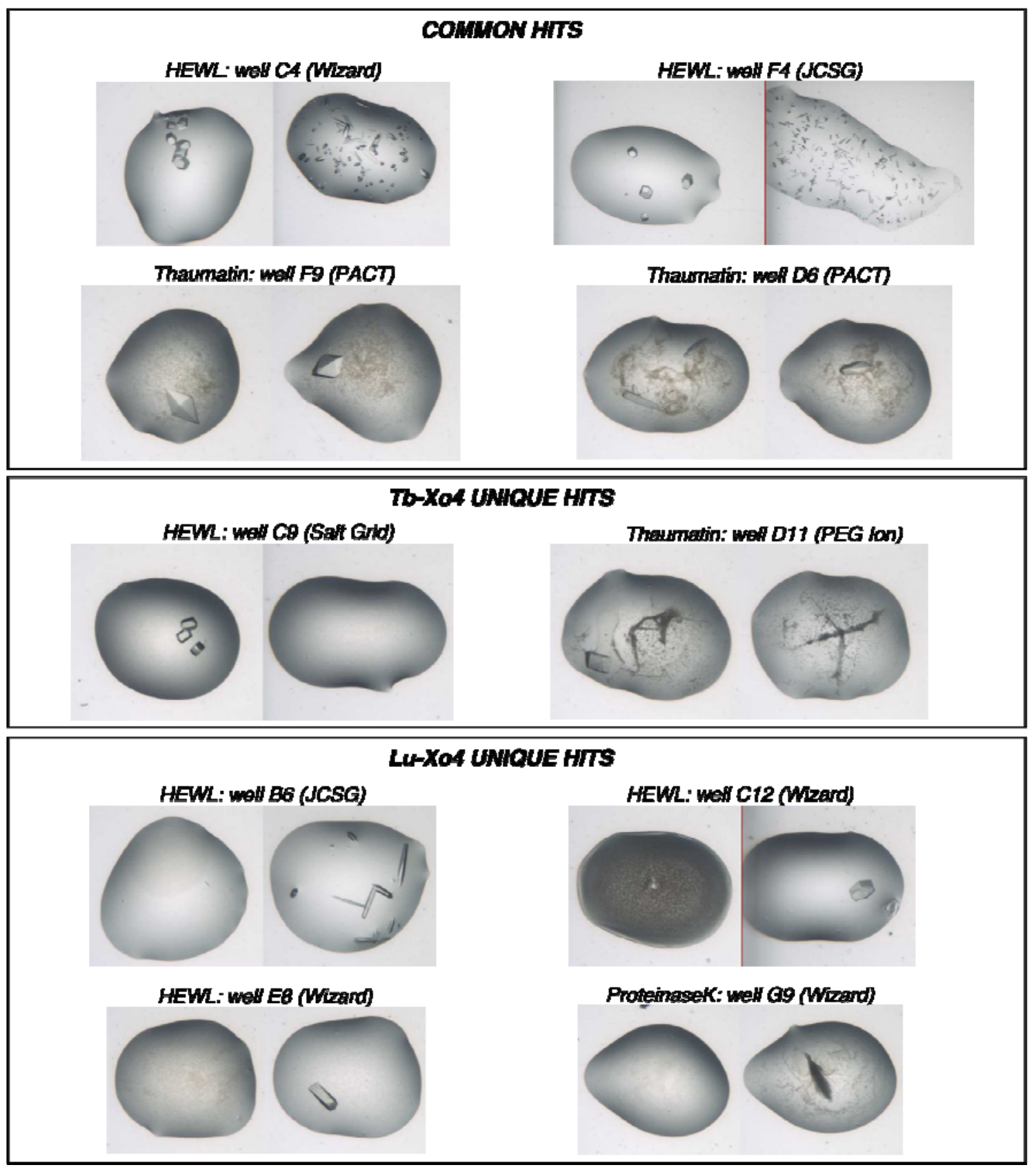
Figure 3: Comparison between the crystallization assays performed with Tb-Xo4 and Lu-Xo4 variants at $10 \mathrm{mM}$ concentration. (a) By-plate representations where hits with $\mathrm{Tb}-\mathrm{Xo} 4$ are depicted by a circle while hits obtained in the presence of Lu-Xo4 are represented with a backslash. (b) Examples of crystallization drops observed in common crystallization conditions (left drops: Tb-Xo4; right drops: Lu-Xo4), in conditions where only Tb-Xo4 leads to crystals and in conditions where crystals only appear in the presence of Lu-Xo4.

Subset-of-interest representation and exploitation. We also proposed a second way to represent results of crystallization assays. It consists in breaking the by-plate level through the definition of a new space called subset-of-interest (SOI), similarly to the notion of region-ofinterest used in image processing for example. In this representation (Figure 4), each crystallization condition leading to a hit is represented by a square. All hit crystallization conditions, for both native and crystallophore assays, are sort out and shared in three categories $(-1,0$, or +1$)$ depending of the effect of the crystallophore: $(0)$ the protein crystallizes with and without the crystallophore (it does not affect crystallization), (-1) the protein crystallizes only in absence of the additive ( $\mathrm{Tb}-\mathrm{Xo} 4$ has a negative effect on the crystallization), and $(+1)$ the protein crystallizes only in the presence of the complex ( $\mathrm{Tb}-\mathrm{Xo} 4$ has a positive effect). At this stage, the SOI representation could be viewed as an improvement of the histogram representation but it provides a more powerful way to analyze the data as illustrated hereafter.

The presence of a specific parameter can be indicated to highlight possible synergetic or antagonist effects with the additive. Graphically, this can be viewed as a layer on the considered representation (Figure 4) and can be used both in by-plate or SOI representations. Examples of parameter to be evaluated includes crystallization kit, $\mathrm{pH}$ or any crystallization component. In the SOI representation, a given parameter more represented in the -1 area means a detrimental effect with the crystallophore while on the contrary, elements appearing in the +1 will indicate a potential synergetic effect.

Figure 4 illustrates this with two examples of parameters at a single protein level. When $\mathrm{pH}$ is considered as parameter, more hits with crystallization solutions with $\mathrm{pH}$ above 7 are observed for both proteins in the presence of $\mathrm{Tb}-\mathrm{Xo} 4$ compared to the protein alone (Figure 4a). In particular, the $\mathrm{pH}=9-10$ conditions appear only in the +1 area in the case of ProteinaseK clearly indicating a more favorable nucleating condition for the protein in the presence of $\mathrm{Tb}-\mathrm{Xo} 4$ in 
such basic medium. We also look at the potential synergy between the crystallophore and a given commercial crystallization kit (Figure 4b). For ProteinaseK when Tb-Xo4 is added, we observed a noticeable increase of the number of hits related to conditions present in the PEGs-Ion kit from Qiagen. A similar synergy with the crystallophore is present for this kit in the case of pb9 as well as an increase of the hits associated with the JCSG kit (Molecular Dimensions). Such analysis are obviously protein-dependent and may be of interest for any future user of crystallophore to determine the best physico-chemical conditions for their protein target to be crystallized. This kind of analysis can also be applied to any other nucleating agents.

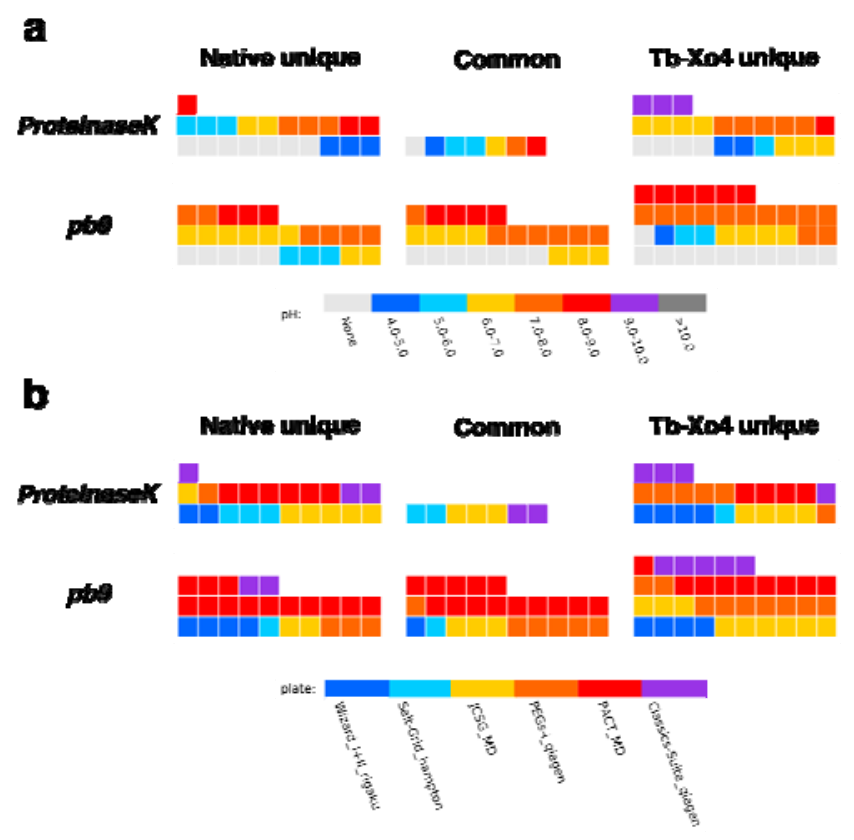

Figure 4: SOI representation of the analysis of the influence of (a) $\mathrm{pH}$ and (b) crystallization kit on the crystallization of Proteinase $\mathrm{K}$ and $\mathrm{pb} 9$ in the absence/presence of $10 \mathrm{mM} \mathrm{Tb}-\mathrm{Xo} 4$. Each square represents a crystallization hit. The three area of the SOI correspond to the hits observed for the protein in the absence/presence of Tb-Xo4 (-1/+1 area, respectively) and observed in both cases ( 0 area). The reported $\mathrm{pH}$ value corresponds to the value of the crystallization conditions available in the description of the commercial kits. None indicates that no value is provided in the description.

However, as part of our ambition to understand the nucleating properties of the crystallophore a larger data set should be considered to ensure meaningful results. The SOI analysis shows its 
full interest when comparative crystallization results of the 6 proteins are taken into account as illustrated in Figure 5. We again considered $\mathrm{pH}$ and crystallization kits as parameters.
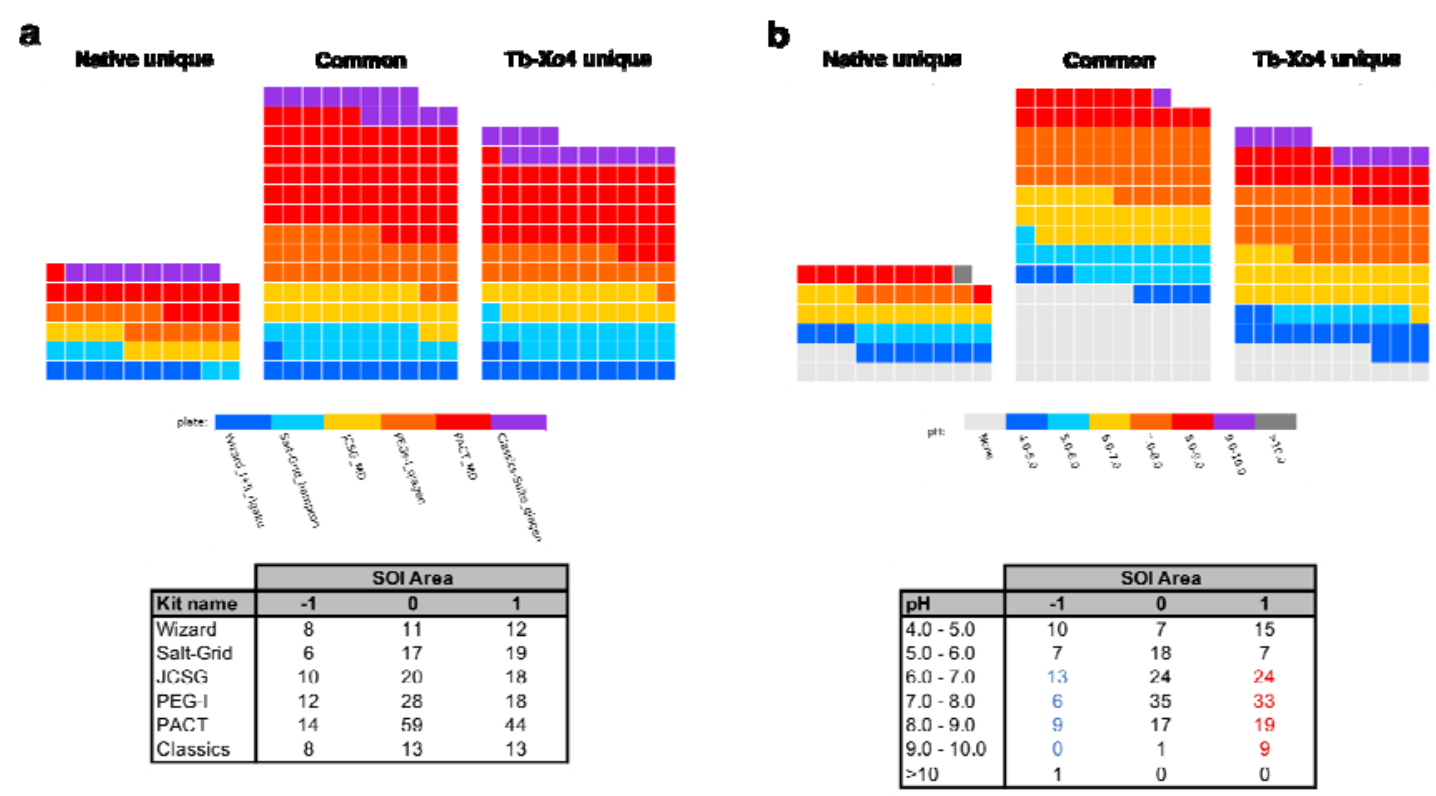

Figure 5: SOI representation and analysis (Tables) of the crystallization assays of the 6 considered proteins relative to (a) commercial crystallization kit and (b) $\mathrm{pH}$. Each square represents a crystallization hit. The three areas of the SOI correspond to the hits observed for the protein in the absence/presence of $\mathrm{Tb}-\mathrm{Xo} 4(-1 /+1$ area, respectively) and observed in both cases ( 0 area). The reported $\mathrm{pH}$ value corresponds to the value of the crystallization conditions available in the description of the commercial kits. None indicates that no value is provided in the description.

Compared to the conclusion drawn in the case of ProteinaseK and pb9, the analysis performed on the 6 proteins shows a different result on a potential synergy between the crystallophore and a given crystallization kit. We observed a clear increase of the hit number with both Salt-Grid and PACT kit with 3 times more hits when Tb-Xo4 is present (19 and 44 crystallization hits, 
respectively) compared to in the absence of the crystallophore (Figure 5a). With the basic aim to limit the number of conditions to be evaluated, the analysis performed on the potential synergy between the crystallophore and commercial kits (Figure 5a) shows that the PACT crystallization kit ${ }^{36}$ maybe a good starting point. Our foreseen studies will obviously focus on the influence of each crystallization components such buffer composition, precipitant nature (PEGs, salts...) or the presence of anions or cations in the crystallization media. Indeed thanks to crystal structure analysis of Tb-Xo4 interactions at the protein surface, ${ }^{28}$ we have observed that components of crystallization solutions, such as sulfate, iodide or magnesium, may directly be involved in the interaction.

The $\mathrm{pH}$ tendency observed with ProteinaseK and pb9 proteins is confirmed. More hits (red values in the Table, Figure 5b) are obtained in the presence of $\mathrm{Tb}-\mathrm{Xo} 4$ when the $\mathrm{pH}$ of the crystallization solution is in the range 6-10 compared to native-only hits (blue values in the Table, Figure 5b). The design of Xo4 was done in order to target aspartate and glutamate residues as $\mathrm{Tb}-\mathrm{Xo} 4$ is a cationic complex. This was later confirmed through crystal structure analysis of Xo4 binding showing a direct coordination of the lanthanide ion by acidic residues, ${ }^{28}$ even though other binding modes were described. Our preliminary observation on the effect of $\mathrm{pH}$ are in line with the supposed importance of Xo4 direct coordination. Indeed we observed that the crystallophore favors crystal appearance in crystallization conditions with a $\mathrm{pH}$ ranging between 6 and 10. Such pH values ensure that the carboxylate moiety of both aspartate and glutamate are fully deprotonated to ensure a direct interaction with the lanthanide ion(III). This illustrates the added value of setting-up such database. To better take into account the effect of $\mathrm{pH}$ and because a non-negligible portion of the commercially available crystallization solutions are provided without any associated $\mathrm{pH}$ information, one should get the $\mathrm{pH}$ value of each solution by direct measurement or through the use of a dye-based assay as implemented at the CSIRO Collaborative Crystallization Centre (C3). ${ }^{37}$ It would also be of interest to look at correlation between crystallophore nucleating properties and the characteristics of considered proteins such their isoelectric point, the proportion of acidic residues or the ratio between acidic/basic amino acids. For that we plan to introduce a fourth table within the database structure that will contain protein information as depicted in Table 1. 


\section{CONCLUSION}

In this article, we present the structure of a database aimed at facilitating the understanding of the unique nucleating properties of a class of lanthanide complexes, the crystallophore. The database exploitation is facilitated by associated tools such as the different representations that have been exemplified. In particular, the concept of subset-of-interest has been introduced to reveal potential antagonistic/synergistic effects between Xo4 and other physico-chemical parameters such as $\mathrm{pH}$. The overall approach may be of interest for any studies working on solutions dedicated to improve the nucleating step in protein crystallization.

Thanks to the object-oriented nature of the Python language, as well as the huge amount of available programming libraries, the future development of the database is straight-forward, whether into a stand-alone package or a web-based tool. Finally, the pertinence of such statistical analysis requires that the database is fed with enough occurrences and we invite any users of the crystallophore to share with us the results of their comparative crystallization assays.

\section{Author Contributions}

The manuscript was written through contributions of all authors. All authors have given approval to the final version of the manuscript.

\section{Funding Sources}

This work was supported by the French Agence Nationale de la Recherche (ANR) through the project Ln23 (ANR-13-BS07-0007-01), by the Region Auvergne-Rhône-Alpes through the project Xo4-2.0 (Pack Ambition Recherche 2017) and by the Fondation Maison de la Chimie.

\section{Notes}

OM, FR, EG and SE declare a potential conflict of interest since they are co-founders of the Polyvalan company that commercializes the crystallophore.

\section{ACKNOWLEDGMENT}

IBS acknowledges integration into the Interdisciplinary Research Institute of Grenoble (IRIG, CEA). This work used the platforms of the Grenoble Instruct-ERIC center (ISBG; UMS 3518 
CNRS-CEA-UGA-EMBL) within the Grenoble Partnership for Structural Biology (PSB), supported by FRISBI (ANR-10-INBS-05-02) and GRAL, financed within the University Grenoble Alpes graduate school (Ecoles Universitaires de Recherche) CBH-EUR-GS (ANR-17EURE-0003). Authors also thank the pôle de compétitivité Lyon-Biopôle. Authors are grateful to Christèle Maury for fruitful discussion in the elaboration of the SOI analysis.

\section{ABBREVIATIONS}

Xo4, crystallophore; SOI, subset-of-interest; HEWL, Hen egg white lysozyme; GRHPR, Glyoxylate Hydroxypyruvate Reductase.

\section{REFERENCES}

(1) Ireton, G. C.; Stoddard, B. L. Microseed Matrix Screening to Improve Crystals of Yeast Cytosine Deaminase. Acta Crystallogr D Biol Crystallogr 2004, 60 (Pt 3), 601-605.

(2) D'Arcy, A.; Villard, F.; Marsh, M. An Automated Microseed Matrix-Screening Method for Protein Crystallization. Acta Crystallogr D Biol Crystallogr 2007, 63 (Pt 4), 550-554.

(3) Shaw Stewart, P. D.; Kolek, S. A.; Briggs, R. A.; Chayen, N. E.; Baldock, P. F. M. Random Microseeding: a Theoretical and Practical Exploration of Seed Stability and Seeding Techniques for Successful Protein Crystallization. Crystal Growth \& Design 2011, 11 (8), 34323441.

(4) Obmolova, G.; Malia, T. J.; Teplyakov, A.; Sweet, R. W.; Gilliland, G. L. Protein Crystallization with Microseed Matrix Screening: Application to Human Germline Antibody Fabs. Acta Crystallogr F Struct Biol Commun 2014, 70 (Pt 8), 1107-1115.

(5) McPherson, A.; Shlichta, P. Heterogeneous and Epitaxial Nucleation of Protein Crystals on Mineral Surfaces. Science 1988, 239 (4838), 385-387.

(6) Chayen, N. E.; Saridakis, E.; El-Bahar, R.; Nemirovsky, Y. Porous Silicon: an Effective Nucleation-Inducing Material for Protein Crystallization. J Mol Biol 2001, 312 (4), 591-595. 
(7) D'Arcy, A.; Mac Sweeney, A.; Haber, A. Using Natural Seeding Material to Generate Nucleation in Protein Crystallization Experiments. Acta Crystallogr D Biol Crystallogr 2003, 59 (Pt 7), 1343-1346.

(8) Saridakis, E.; Khurshid, S.; Govada, L.; Phan, Q.; Hawkins, D.; Crichlow, G. V.; Lolis, E.; Reddy, S. M.; Chayen, N. E. Protein Crystallization Facilitated by Molecularly Imprinted Polymers. Proceedings of the National Academy of Sciences 2011, 108 (27), 11081-11086.

(9) Khurshid, S.; Saridakis, E.; Govada, L.; Chayen, N. E. Porous Nucleating Agents for Protein Crystallization. Nat Protoc 2014, 9 (7), 1621-1633.

(10) Leese, H. S.; Govada, L.; Saridakis, E.; Khurshid, S.; Menzel, R.; Morishita, T.; Clancy, A. J.; White, E. R.; Chayen, N. E.; Shaffer, M. S. P. Reductively PEGylated Carbon Nanomaterials and Their Use to Nucleate 3D Protein Crystals: a Comparison of Dimensionality. Chem. Sci. 2016, 7 (4), 2916-2923.

(11) Chen, Y.-W.; Lee, C.-H.; Wang, Y.-L.; Li, T.-L.; Chang, H.-C. Nanodiamonds as Nucleating Agents for Protein Crystallization. Langmuir 2017, 33 (26), 6521-6527.

(12) Pompidor, G.; D'Aléo, A.; Vicat, J.; Toupet, L.; Giraud, N.; Kahn, R.; Maury, O. Protein Crystallography Through Supramolecular Interactions Between a Lanthanide Complex and Arginine. Angewandte Chemie International Edition 2008, 47 (18), 3388-3391.

(13) Bijelic, A.; Rompel, A. The Use of Polyoxometalates in Protein Crystallography - an Attempt to Widen a Well-Known Bottleneck. Coord Chem Rev 2015, 299, 22-38.

(14) Molitor, C.; Bijelic, A.; Rompel, A. The Potential of Hexatungstotellurate(VI) to Induce a Significant Entropic Gain During Protein Crystallization. IUCrJ 2017, 4 (Pt 6), 734-740.

(15) Alex, J. M.; Rennie, M. L.; Volpi, S.; Sansone, F.; Casnati, A.; Crowley, P. B. Phosphonated Calixarene as a "Molecular Glue" for Protein Crystallization. Crystal Growth \& Design 2018, 18 (4), 2467-2473. 
(16) Alex, J. M.; Engilberge, S.; Fizil, Á.; Rennie, M. L.; Lehoczki, G.; Dorottya, H.; Batta, G.; Crowley, P. B. Calixarene-Mediated Assembly of a Small Antifungal Protein. IUCrJ 2019, 6 (2), 238-247.

(17) Engilberge, S.; Rennie, M. L.; Dumont, E.; Crowley, P. B. Tuning Protein Frameworks via Auxiliary Supramolecular Interactions. ACS Nano 2019, 13 (9), 10343-10350.

(18) Engilberge, S.; Riobé, F.; Di Pietro, S.; Lassalle, L.; Coquelle, N.; Arnaud, C.-A.; Pitrat, D.; Mulatier, J.-C.; Madern, D.; Breyton, C.; Maury, O.; Girard, E. Crystallophore: a Versatile Lanthanide Complex for Protein Crystallography Combining Nucleating Effects, Phasing Properties, and Luminescence. Chem. Sci. 2017, 8 (9), 5909-5917.

(19) Engilberge, S.; Wagner, T.; Santoni, G.; Breyton, C.; Shima, S.; Franzetti, B.; Riobé, F.; Maury, O.; Girard, E. Protein Crystal Structure Determination with the Crystallophore, a Nucleating and Phasing Agent. Journal of Applied Crystallography 2019, 52 (Pt 4), 722-731.

(20) Vögeli, B.; Engilberge, S.; Girard, E.; Riobé, F.; Maury, O.; Erb, T. J.; Shima, S.; Wagner, T. Archaeal Acetoacetyl-CoA Thiolase/HMG-CoA Synthase Complex Channels the Intermediate via a Fused CoA-Binding Site. Proceedings of the National Academy of Sciences 2018, 115 (13), 3380-3385.

(21) Bernhardsgrütter, I.; Vögeli, B.; Wagner, T.; Peter, D. M.; Cortina, N. S.; Kahnt, J.; Bange, G.; Engilberge, S.; Girard, E.; Riobé, F.; Maury, O.; Shima, S.; Zarzycki, J.; Erb, T. J. The Multicatalytic Compartment of Propionyl-CoA Synthase Sequesters a Toxic Metabolite. Nature Chemical Biology 2018, 14 (12), 1127-1132.

(22) Roche, J.; Girard, E.; Mas, C.; Madern, D. The Archaeal LDH-Like Malate Dehydrogenase From Ignicoccus Islandicus Displays Dual Substrate Recognition, Hidden Allostery and a Non-Canonical Tetrameric Oligomeric Organization. J Struct Biol 2019, 208 (1), $7-17$.

(23) Rempel, S.; Colucci, E.; de Gier, J. W.; Guskov, A.; Slotboom, D. J. Cysteine-Mediated Decyanation of Vitamin B12 by the Predicted Membrane Transporter BtuM. Nat Commun 2018, $9(1), 3038$. 
(24) Hajj Chehade, M.; Pelosi, L.; Fyfe, C. D.; Loiseau, L.; Rascalou, B.; Brugière, S.; Kazemzadeh, K.; Vo, C.-D.-T.; Ciccone, L.; Aussel, L.; Couté, Y.; Fontecave, M.; Barras, F.; Lombard, M.; Pierrel, F. A Soluble Metabolon Synthesizes the Isoprenoid Lipid Ubiquinone. Cell Chem Biol 2019, 26 (4), 482-492.e487.

(25) Schada von Borzyskowski, L.; Severi, F.; Krüger, K.; Hermann, L.; Gilardet, A.; Sippel, F.; Pommerenke, B.; Claus, P.; Cortina, N. S.; Glatter, T.; Zauner, S.; Zarzycki, J.; Fuchs, B. M.; Bremer, E.; Maier, U. G.; Amann, R. I.; Erb, T. J. Marine Proteobacteria Metabolize Glycolate via the B-Hydroxyaspartate Cycle. Nature 2019, 575 (7783), 500-504.

(26) Kumari Yadav, R.; Krishnan, V. The Adhesive PitA Pilus Protein From the Early Dental Plaque Colonizer Streptococcus Oralis: Expression, Purification, Crystallization and X-Ray Diffraction Analysis. Acta Crystallogr F Struct Biol Commun 2020, 76 (Pt 1), 8-13.

(27) Belot, L.; Ouldali, M.; Roche, S.; Legrand, P.; Gaudin, Y.; Albertini, A. A. Crystal Structure of Mokola Virus Glycoprotein in Its Post-Fusion Conformation. PLoS Pathog. 2020, $16(3), \mathrm{e} 1008383$.

(28) Engilberge, S.; Riobé, F.; Wagner, T.; Di Pietro, S.; Breyton, C.; Franzetti, B.; Shima, S.; Girard, E.; Dumont, E.; Maury, O. Unveiling the Binding Modes of the Crystallophore, a Terbium-Based Nucleating and Phasing Molecular Agent for Protein Crystallography. Chem. Eur. J. 2018, 24 (39), 9739-9746.

(29) Girard, E.; Anelli, P. L.; Vicat, J.; Kahn, R. High-Phasing-Power Lanthanide Derivatives: Taking Advantage of Ytterbium and Lutetium for Optimized Anomalous Diffraction Experiments Using Synchrotron Radiation. Acta Crystallogr D Biol Crystallogr 2003, 59 (Pt 10), 1877-1880.

(30) Fazio, V. J.; Peat, T. S.; Newman, J. A Drunken Search in Crystallization Space. Acta Crystallogr F Struct Biol Commun 2014, 70 (Pt 10), 1303-1311.

(31) Gorrec, F. Protein Crystallization Screens Developed at the MRC Laboratory of Molecular Biology. Drug Discov Today 2016, 21 (5), 819-825. 
(32) Pereira, J. H.; McAndrew, R. P.; Tomaleri, G. P.; Adams, P. D. Berkeley Screen: a Set of 96 Solutions for General Macromolecular Crystallization. Journal of Applied Crystallography 2017, 50 (Pt 5), 1352-1358.

(33) Kirkwood, J.; Hargreaves, D.; O'Keefe, S.; Wilson, J. Analysis of Crystallization Data in the Protein Data Bank. Acta Crystallogr F Struct Biol Commun 2015, 71 (Pt 10), 1228-1234.

(34) Ng, J. T.; Dekker, C.; Reardon, P.; Delft, von, F. Lessons From Ten Years of Crystallization Experiments at the SGC. Acta Crystallogr D Struct Biol 2016, 72 (Pt 2), 224235.

(35) Rosa, N.; Ristic, M.; Thorburn, L.; Abrahams, G. J.; Marshall, B.; Watkins, C. J.; Kruger, A.; Khassapov, A.; Newman, J. Tools to Ease the Choice and Design of Protein Crystallisation Experiments. Crystals 2020, 10 (2), 95.

(36) Newman, J.; Egan, D.; Walter, T. S.; Meged, R.; Berry, I.; Ben Jelloul, M.; Sussman, J. L.; Stuart, D. I.; Perrakis, A. Towards Rationalization of Crystallization Screening for Small- to Medium-Sized Academic Laboratories: the PACT/JCSG+ Strategy. Acta Crystallogr D Biol Crystallogr 2005, 61 (Pt 10), 1426-1431.

(37) Newman, J.; Sayle, R. A.; Fazio, V. J. A Universal Indicator Dye pH Assay for Crystallization Solutions and Other High-Throughput Applications. Acta Crystallogr D Biol Crystallogr 2012, 68 (Pt 8), 1003-1009. 